\title{
SUPPORTING THE DESIGN PROCESS BY AN INTEGRATED KNOWLEDGE BASED DESIGN SYSTEM
}

\author{
HANS GRABOWSKI, RALF-STEFAN LOSSACK AND CLEMENS WEIS \\ University of Karlsruhe, Germany
}

\begin{abstract}
The German design methodology demonstrated its usefulness for solving design problems by being applied in the enterprises' design departments during the years. Design methodologists as Roth, Pahl, Beitz and Hubka developed an instrument for a methodological approach to design tasks. This approach is a strongly process oriented one and describes together with the fundamentals of design, general strategies for solving design problems. Another approach, which was followed by the researchers of design systems was an information oriented one. Here the main work was concentrated on the modeling of the information needed in design. There was no approach which combined consequently these two different approaches. In this article we introduce in the first part the fundamental aspects of the German design methodology by describing the modeling space of design with the help of an example of mechanical engineering. In the second part we introduce design working spaces which help to structure and administer design solutions. Finally an approach to incorporate the process oriented aspect of design into a knowledge based CAD system is presented.
\end{abstract}

\section{Introduction and Overview of the Design Process}

Design methodology considers the design process in an idealized manner as a successive concretion of the description of the to-be characteristics of a technical object (Koller, 1985; Pahl and Beitz, 1994; Roth, 1994 and others). This concretion process takes place on the product modeling level. Koller, Pahl, Beitz, Roth and other design methodologists define this process to lead from

- The incomplete to the complete,

- The abstract to the concrete,

- The rough to the precise,

- The provisional to the definitive and

- Possible alternatives to the optimal solution.

An important characteristic of this process is the successive growth of the set of design characteristics with respect to the current state of the design. Here design characteristics are defined as the instantiated solution properties of a product to be developed. The sum of all these solution properties of a 
product characterises in connection with the corresponding product model the properties and the overall behaviour of the product in real life.

The description of the design characteristics mentioned above is the result of the design process. They can be assigned to the design phases known from the design methodology. The design phases consist of defining the requirements of a product, of the definition of the functional structure and the function flow within a product, the description of the physical effects which can be assigned to the respective functions in correspondence with the modeling of the product's effective geometry and the design of the product's shape. From the methodological point of view of design the origin for the phase orientation of the design process is found here. In accordance to these phases the logical modeling layers of a design system have been defined as follows:

\subsection{REQUIREMENTS MODELLING LAYER}

The requirements modeling layer serves for the computational projection of the results won by the clarification of the design task. This contains the preconditions of the design, the definition of product requirements, i.e. the to-be properties of the future product and the description of the product's immanent task structure as the transition to the functional modeling.

\subsection{FUNCTIONAL MODELLING LAYER}

Functional modeling serves for finding and describing the functions of a design solution to be developed, as well as the functional interrelationships within the future product. Functional modeling allows the definition and manipulation of functions on different levels of abstraction, as well as the description of their interrelations in functional structures. Another aspect which is of importance for the course of the design process is the functional flow within the product under development. The logical transition and by that the concretion (described later) of the functional model to the conceptual model is supported by the specification of the vectorial functional structure in accordance with defining the corresponding design working spaces.

\subsection{CONCEPTUAL DESIGN MODELING LAYER}

The conceptual design serves for the description of the solution concept of a design task. It covers all information fixed while describing the product's physical solution principles. These information contain the physical effects used for solving the problem in correspondence with the mathematical equations describing them. Geometrical information as e. g. effective lines, effective faces and effective spaces are also modeled within the conceptual 
design. This phase is completed by assigning the concepts modeled to the functions of the functional structure and by grouping the concepts into the conceptual structure.

\subsection{SHAPE MODELLING LAYER}

The shape modeling is the most concrete of the product modeling layers. For that it completes the product modeling process by the geometrical definition of all design features and design working spaces to completely defined three dimensional parts with assigned material and their combination to groups and group structures.

\section{The Modeling Space of Design}

By representing the design process as a process of concretion performed on different levels of abstraction (which we called in the paragraph above "the modeling layers") it may be misunderstood as a strongly forward oriented process. This is only the case in an idealized manner. In practice design evolves as a highly iterative process where, based on a solution state $\mathrm{SS}_{i}{ }_{i}$, different solution directions can be followed in order to reach a following solution state $\mathrm{SSO}_{\mathrm{i}+1}$. In the following we understand by the term solution state of an object $\mathrm{SS}^{\circ}{ }_{i}$ the instantiation of an object demarcated by the corresponding design working space belonging to the intended product after the i-th design step. Figure 1 gives an idea of this procedure. Starting at any solution state of a design task the solution directions showed can be followed. These solution directions which are derived by Birkhofer (1980), Krumhauer (1975) and Rude (1991) from the modeling space of design describe a possible way to transform a solution state $\mathrm{SS}_{\mathrm{i}}$ into a following $\mathrm{SS}_{\mathrm{i}+1}$. In general this means to be one step closer to the intended solution.

Before stepping into more detail of the fundamentals of the design process we direct some interest on the design shown in Figure 2. It shows the presentation of a robot gripper whose design serves as example for explaining our abstract model of the design process. The robot gripper serves for the handling of small parts. It is designed for durability and for low maintenance costs. A standard connection to the robot arm was given as well as the space in which the gripper has to fit. The working method of the gripper is as follows: The force with which the handled part is gripped originates from the application of a pressure that is foreseen by the robot. The resulting force then is transmitted through a bar to a wedge at the end of the bar. The wedge splits the force into two resulting forces which are applied to the jaws of the gripper. The applied pressure causes a movement of the piston towards the jaws. The wedge at the end of the piston then 
causes a turning motion of the jaws which results in the gripping force of the robot gripper.

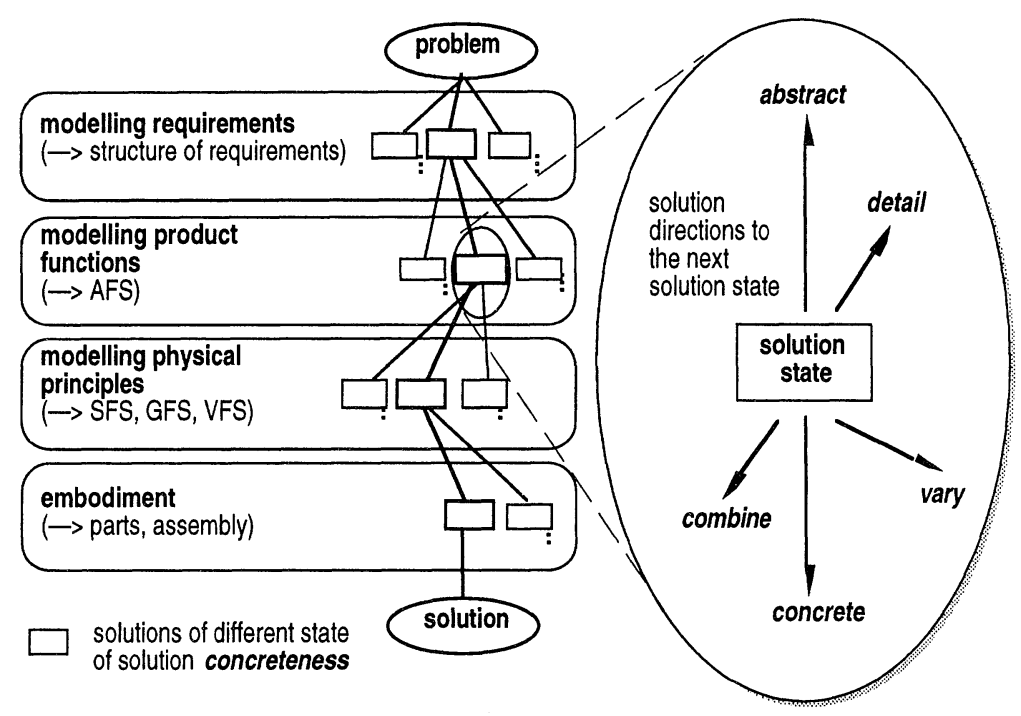

Figure 1. Elementary solution steps in the design process.

The detachment of the handled product is started by reducing the pressure applied on the piston. So a spring which is mounted in the front of the piston pushes it back to its original position. So the gripper's jaws can also be opened by a spring.

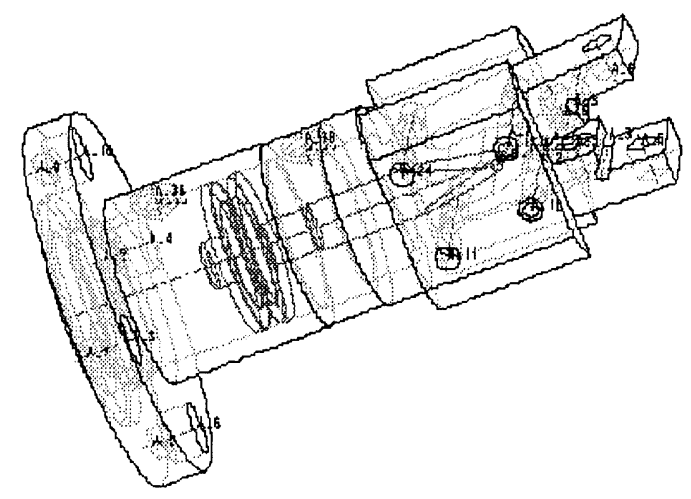

Figure 2. Product example: Robot gripper, which serves as the intended design.

In the following, the solution directions mentioned above are explained with the help of some examples from the design of a robot gripper (for a short description of the symbols used in the example please see section 3 ). 


\subsection{CONCRETION}

The transformation of a solution state $\mathrm{SS}^{\mathrm{O}}{ }_{\mathrm{i}}$ into a following, more concrete one $\mathrm{SS}_{\mathrm{i}+1}$ is called "concretion". Here we understand by the term "more concrete solution state" the instantiation of the product model with information belonging to a more concrete modeling layer (see Figure 3). By this, new solution properties are added to the solution state $\mathrm{SS}_{\mathrm{i}}$. The example shows the concretion of the structure of physical principles of a robot gripper on the functional modeling layer to a conceptual design sketch on the conceptual design level.

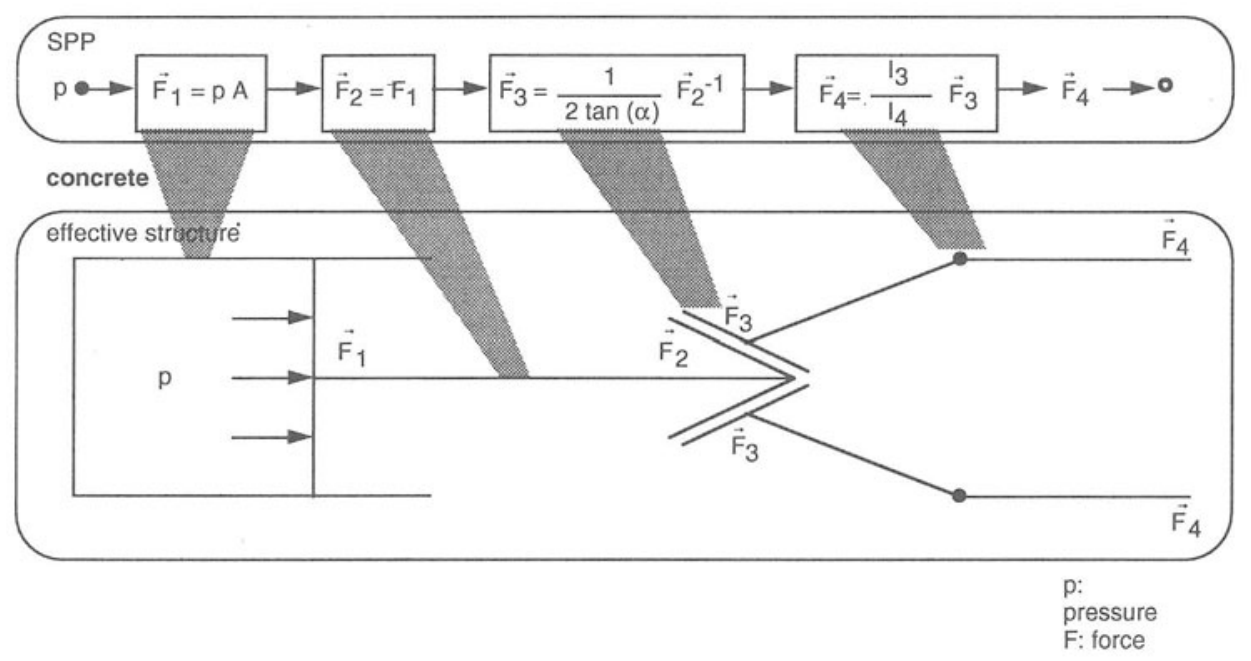

Figure 3. Example for the solution direction "concretion".

In our example the structure of physical principles (SPP) is concreted onto the effective structure of the intended robot gripper. The physical principle which describes the transformation of a pressure into a force $\left(F_{1}=\right.$ $\mathrm{pA}$ ) is mapped to the effective structure of a pressure cylinder with a piston inside. The transmittance of the force $\left(F_{2}=F_{1}\right)$ is realized by a bar which is attached to the piston. A wedge at the end of the piston splits the force into two effective forces and changes their directions $\left(F_{3}=(1 / 2 \tan (\alpha)) F_{2}^{-1}\right) . F_{4}$, which is the force responsible for the gripping action, is obtained by leading $\mathrm{F}_{3}$ into a lever. With that a rough sketch of the effective structure is obtained. This sketch is the basis for the next design steps.

\subsection{ABSTRACTION}

The solution direction "abstraction" is opposite to the direction concretion. This means the solution state $\mathrm{SS}_{\mathrm{i}}$ is transformed with respect to the intended 
design solution into a more abstract solution state $\mathrm{SS}_{\mathrm{i}+1}$. Abstraction serves for the recognition of essential product properties. In consequence the more abstract solution state $\mathrm{SS}^{\mathrm{O}}{ }_{\mathrm{i}+1}$ is one step further away from the intended design solution (see Figure 4). This step can be used for starting from known designs to reach new until then not known solutions. In this context Figure 4 shows the abstraction of the shape of a robot gripper's jaw onto its effective structure.
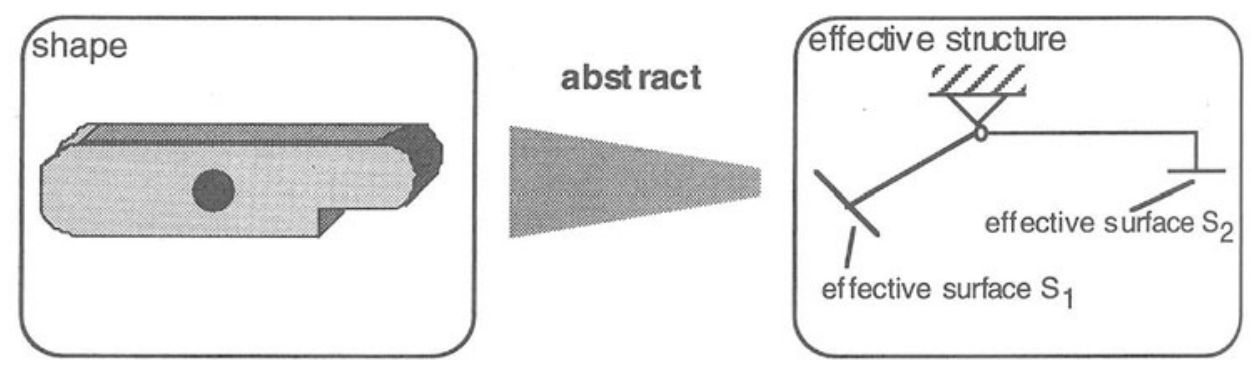

Figure 4. Example for the solution direction "abstraction".

Starting from the shape of the robot gripper's jaw, the essential features of the design are extracted. These are the two effective surfaces $S_{1}$ and $S_{2}$ where $S_{1}$ is responsible for the transmittance of a force onto the lever, which can also be extracted from the shape. $S_{2}$ is the effective surface which can be recognized as being responsible for applying the gripping force onto the handled part. The function of the hole in the middle of the jaw's body acts as a bearing which also finds its counterpart in the effective structure. By that the shape description of the robot gripper's jaw is abstracted to its effective structure. The effective structure can serve as the basis for a variation of the shape or it can be abstracted by itself in order to obtain another effective structure with other design properties.

\subsection{DETAILING}

Adding more information to a design object within the same modeling layer is called detailing. When detailing a solution state $\mathrm{SS}_{i}$ to a following $\mathrm{SS}_{i+1}$, the modeled design information remains on the same level of abstraction as it was in state $\mathrm{SS}_{\mathrm{i}}$. The solution direction detailing is used in order to solve a design problem by dividing it into sub-problems. Figure 5 shows an example for the solution direction detailing. The rough effective structure sketch is detailed by adding two bearings to the two levers and to the bar with the function "to channel force", by adding a sealing to the piston and by designing the effective surfaces which are responsible for applying the gripping force to the part to be handled. So the effective structure of the 
robot gripper now contains more information than in the solution state before but the information contained in the model is still remaining on the same level of abstraction (effective structure). With all the information contained in the detailed sketch it is easier to concrete (maybe after performing other detailing steps) the effective structure to the robot gripper's shape model.

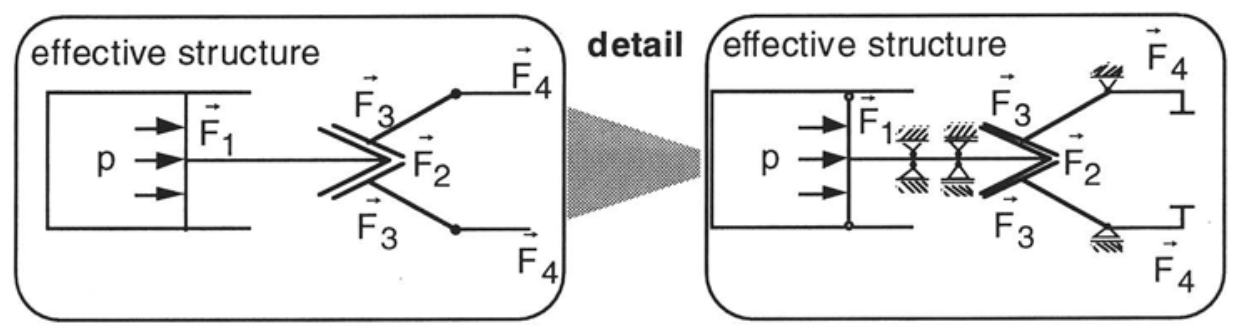

Figure 5. Example for the solution direction "detailing".

\subsection{COMBINATION}

This direction in the model space of design is opposite to detailing. It transforms a solution state $\mathrm{SS}_{\mathrm{i}}$ into a more general one $\mathrm{SS}_{\mathrm{i}+1} . \mathrm{SS}_{\mathrm{i}+1}$ in this case describes a summary of solution properties of the preceding solution state. One example for combination is to summarize different sub-functions into an overall function or, as shown in Figure 6, to omit different design objects in a conceptual design sketch in order to find the basis for a better variant of the intended product. Combination is also the basis for the "abstraction" direction in the modeling space of design. A design on a certain level of abstraction is combined until only the information which is essential for the next design step (abstraction or variation) is left. In our example the combined effective structure of the robot gripper's body may serve as a basis for abstracting it to its functional structure (e. g. structure of physical principles).

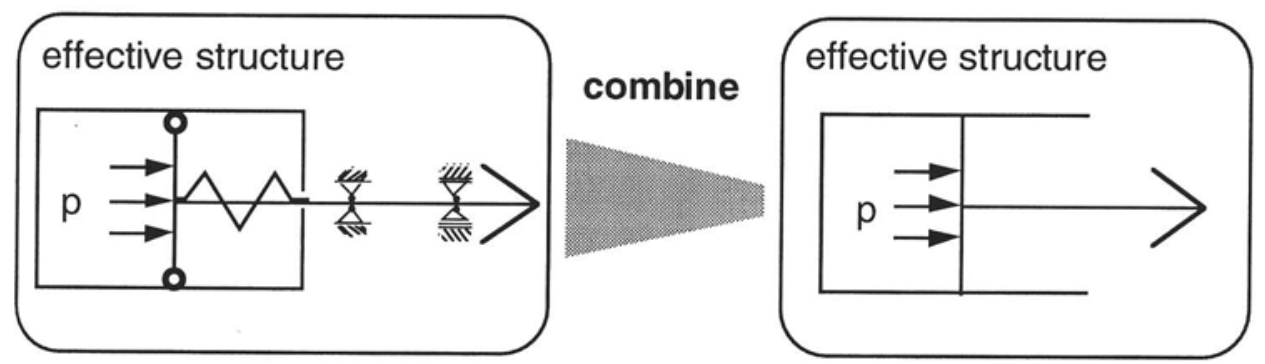

Figure 6. Example for the solution direction "combination". 


\subsection{VARIATION}

Variation means to find to a corresponding solution, in state $\mathrm{SS}_{\mathrm{i}}$ of the intended design, other eventually better alternatives (solution state $\mathrm{SS}^{\mathrm{O}}{ }_{\mathrm{i}+1}$ ). Variation steps back to the preceding solution state $\mathrm{SS}_{\mathrm{i}-1}$ and concretes this solution state to $\mathrm{SSO}_{i+1}$. $\mathrm{SS}_{i+1}$ contains design properties different from the one's of $\mathrm{SS}^{\mathrm{O}}{ }_{\mathrm{i}}$. Variation keeps the intended design's degree of concretion unchanged. What changes, is the spectrum of possible solutions on the same level of abstraction where the design is in state $\mathrm{SS}_{\mathrm{i}}$. As an example for the here described solution direction, the variation of a physical effect corresponding to a certain sub-function, can be mentioned. Figure 7 shows the variation of the function "to generate an energy" into two variants. One is to obtain a force by applying a pressure $\mathrm{p}\left(\mathrm{F}_{1}=\mathrm{f}^{1}(\mathrm{p})\right)$, the other variant is to obtain the force by inserting a spring $\left(F_{1}{ }^{\prime}=f^{1^{\prime}}(D)\right)$, where $D$ is the elasticity constant of the spring.

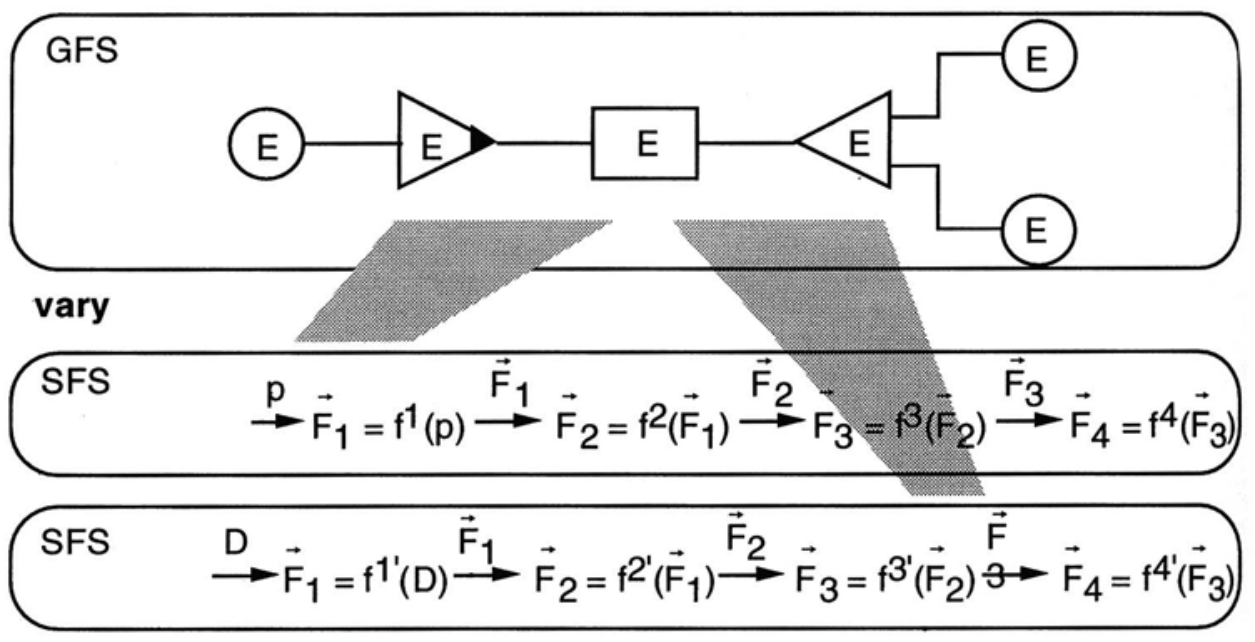

Figure 7. Example for the solution direction "variation".

\subsection{GENERAL PROBLEM SOLVING CYCLE}

Above we discussed the basic and elementary solution steps in the design process. Proceeding in the discussed directions after each step a problemsolving cycle has to be performed. This approach has been derived by the design methodology from the psychology of problem solving.

Figure 8 shows the basic scheme of this general problem-solving cycle (Rutz, 1985). First the designer (for reasons of better readability in the following we call the person who performs the design process "the designer" even if the term "designer/designeress" would be the more correct one) is confronted with the problem. Afterwards the definition of the 
essential problems is performed by fixing the objectives, main constraints and the environment for the intended solution. The next step is finding and representing a solution for the defined problem (this is the creative part of design). After that the solution has to be evaluated followed by making a decision. For one found alternative on the basis of the found solution's evaluation result. Finally as the following step of the design process, the problem-solving cycle is reiterated. So the established solution serves as a definition for the next problem. In this way, we proceed from the qualitative to the quantitative, from the abstraction to the concretion, from the incomplete to the complete etc. This general problem solving cycle is together with the design methodology, which was described above, the basis for the development of our process model of design.

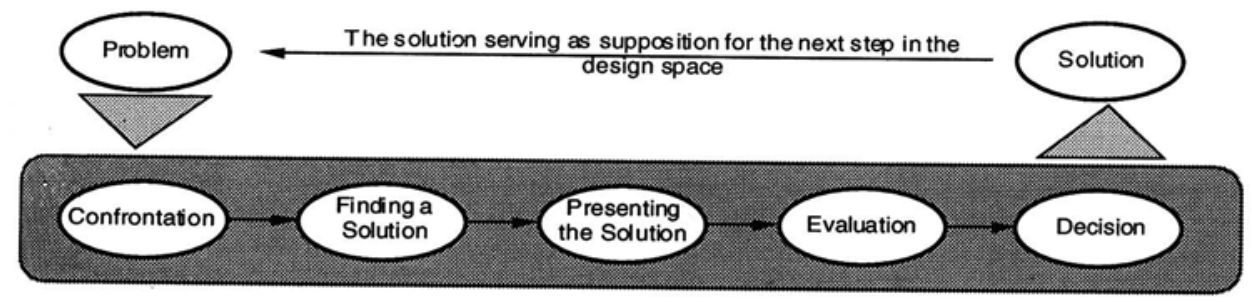

Figure 8. General problem-solving cycle.

\section{Fundamentals of the Design Process for Technical Systems}

With the three fundamental magnitudes of design, matter, energy and information every technical system or artifact can be described on an abstract physical level (Roth, 1994, Pahl and Beitz, 1994). Matter, energy and information are basic concepts (Weizsäcker, 1971). Information determines what has to be done to fulfill a certain purpose. Only with energy technical systems are able to perform any change in nature or in itself. Matter is the stuff, a technical system consists of. It is also the medium in which every process takes place. The human himself is the best example for a technical system. In the first instance he used tools to integrate it in his own technical system, later on he created tools themselves (that means he controlled the matter), in the last centuries he learned to control the energy and in our century, he ruled over the information processes (cybernetics). With respect to being that fundamental every design theory has to be based on this three categorical magnitudes, matter, energy and information.

Technical artifacts are connected to the environment by means of inputs and outputs and can be treated like a system. A system can be divided into sub-systems. What belongs to a particular sub-system is determined by the system boundary. With this approach it is possible to describe every 
technical system at every stage of abstraction. Describing a proposed technical artifact by means of a system consisting of elements, which are grouped by the system boundary related with each other by input and output, we use the term "function" or "product function". If the product function is described on the basis of matter, energy and information as inputs and outputs then we use the term "general function" (GF). If the inputs and outputs represent physical magnitudes like force or torque and the relationship between input and output is described by a physical law, then we use the term "special function" (SF). In the case of a GF the relationship between the input and output is expressed by a limited number of so called function verbs. The function verbs describe the proposed transformation between the input and output. With reference to Roth (1994) we use the set of function verbs "Change, Connect, Channel and Store" for the GF. All technical artifacts are complex constructions, so every artifact can be described by a "general function structure and a special function structure" (GFS, SFS). Because of introducing the above function types and their particular structure the fundamental working principle of abstraction is applicable and therefore a top down approach to the design process is possible.

GFS

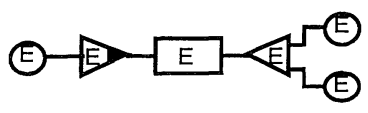

SFS

$\stackrel{p}{\longrightarrow} \vec{F}_{1}=f(p) \stackrel{\vec{F}_{1}}{\longrightarrow} \vec{F}_{2}=f\left(\vec{F}_{1}\right) \stackrel{\vec{r}_{2}}{\longrightarrow} \vec{F}_{3}=k\left(\vec{F}_{2}\right) \stackrel{\vec{F}_{3}}{\longrightarrow} \vec{F}_{4}=f\left(\vec{F}_{3}\right) \stackrel{\vec{F}_{4}}{\longrightarrow}$

SPP

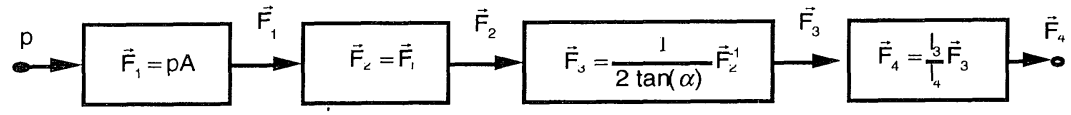

SFS: Special Functionstructur

GFS: General Functionstructur

SPP: Structure of Physical Principles

Figure 9. Established general function structure, its derived special function structure and structure of physical principles.

Figure 9 shows the established General Function Structure (GFS) of the robot gripper of Figure 2, its derived Special Function Structure (SFS) and Structure of Physical Principles (SPP). At the source of the force flow within the intended product there is stored energy depicted by the symbol of a circle containing the character "E". This energy will be changed into another form of energy. On the SFS level there is shown that the energy type 
of pressure will be changed in the energy type of force. After that the energy will be channeled, distributed and amplified. At the bottom of the figure the physical principles which perform this process are shown.

The knowledge about the general functions and special functions and the interrelationship between the different levels of abstraction (or design stages) is modeled in a conceptual object model (Figure 10). Figure 10 is intended to give an idea about the relationships between the objects of the conceptual model In this object model the design knowledge is instantiated. This means the model contains all information which the designer described for the intended product. For that reason the model contains information belonging to all design stages. If all the information described above is contained in the product model on which a design system is based, any technical product can be modeled in this system. But in the product model there is still a lack of information belonging to a mechanism which helps to structure the designed product in order to reduce the complexity of the subtasks. With respect to this problem and also focusing on the administration problem of the subtasks and the corresponding parts of the design we developed the tool of "design working spaces" which is described in the next paragraph.

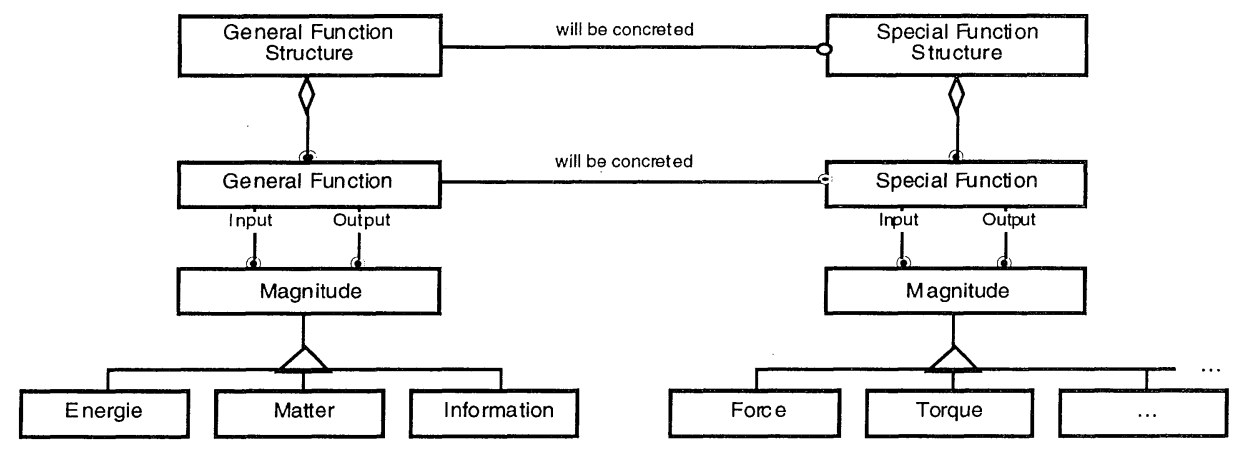

Figure 10. Conceptual object model of general functions, special functions and structures.

\section{Modelling in Design Working Spaces}

A design working space is a Euclidean space (on geometric level, Figure 11) which is available for the designer to solve his design task. The design working space is defined by an envelope (geometric system boundary) and its constraints (inputs/outputs). The fundamental idea of modeling design working spaces comes from system theory and therefore design working spaces are not limited to the geometry. The main purpose of design working 
spaces in this context of modeling design processes is to fix a special design state. If a special design state has been fixed it is possible to derive new design states getting stepwise to the intended solution.

Design working spaces are defined and will be built up by the following rules:

- A design working space consists of a set of elements and of a set of relationships between the elements.

- Elements are a set of information of the design stages, like requirements, product functions or physical principles. Relationships between the elements are general or special magnitudes like energy, information, matter or force, torque etc.

- Every design working space can be subdivided in sub-design working spaces.

- Every element, every design working space and every overall system are inside a given system boundary.

- A system boundary has one or more inputs/outputs and a function which describes the system.

- If a design working space has no inputs/outputs then we talk about a closes design working space on the other hand about an open design working space.
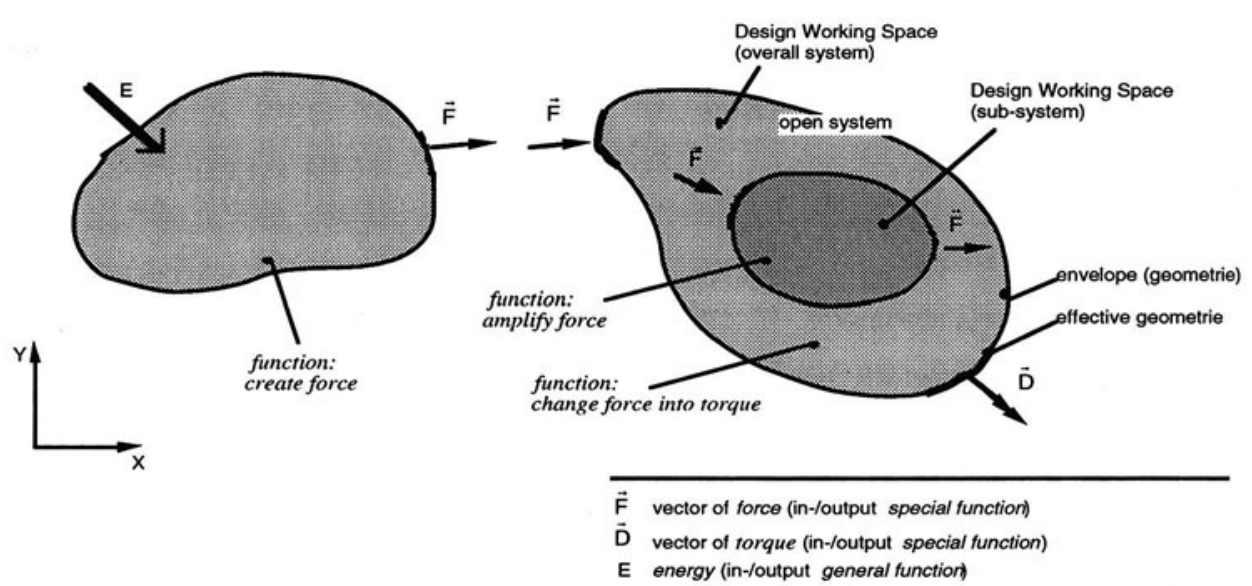

Figure 11. Basic idea of design working spaces.

In Figure 11 there are three design working spaces which have to fulfill a special product function, like create force or amplify force. The design working spaces are clearly defined by their maximum envelope and effective geometry; the envelope and effective geometry is represented by free form surfaces. The envelope describes the maximum space inside which a special 
problem must be solved. The effective geometry is described by effective spaces and effective surfaces which transmit for example forces. The relationship between the design working spaces in Figure 11 is established exemplarily by the general magnitude energy and the special magnitudes force and torque.

As mentioned above in this context the main purpose of design working spaces is to fix a special design state so that new design states can be derived to reach stepwise the intended solution. To derive new design states it is necessary to represent this knowledge in an appropriate model. For this the dynamic model of the design process which is described in the next paragraph, is appropriate. After that in the paragraph of a system architecture the process control model is introduced which represents special state transitions.

\section{An Architecture of a Knowledge Based CAD System Based on the Dynamic Design Process}

The architecture of the knowledge based design system DIICAD ${ }^{1}$ provides four basic components. The purpose of this architecture is oriented towards the mapping of the design methodology described by Pahl/Beitz, Roth, Koller, Hubka and others onto a CAD system.

The first component is the "object model component". It is responsible for the description of the design task as well as for its solutions. All the information describing the intended product is contained in DIICAD's object model (see also paragraph 3). First the requirements, which the product has to fulfil are modeled. This so called "requirement model" then serves as the basis for the following design process (Kläger, 1993). The most abstract level of design is the functional modeling. Herein the functions, a product has to perform, are modeled in a very abstract way. The design methodology provides as the next level of modeling the conceptual design. In conceptual design there are two different views on the product. The first assigns the physical effects to the respective functions of the functional level. The other maps the functions onto the principle structures of the product. The product's effective structure is the result of the conceptual design phase. The functional model of a product in connection with the physical effects and the principle structures form together with the requirements model the basis for the shape design. So all design information needed is stored in the object model.

The second component of the system architecture is the "task solution component". Within this component there are processes defined which transform one solution state $\mathrm{SS}_{i}$ of the design into a following solution state

1 DIICAD: Dialog oriented Integrated Intelligent $\underline{\text { CAD System }}$ 
$\mathrm{SS}_{\mathrm{i}+1}$. Currently there is a case based approach to the task solution component. A learning and self-controlling approach has not yet been attacked. Until now this learning and hopefully self-controlling "component" is the designer himself.

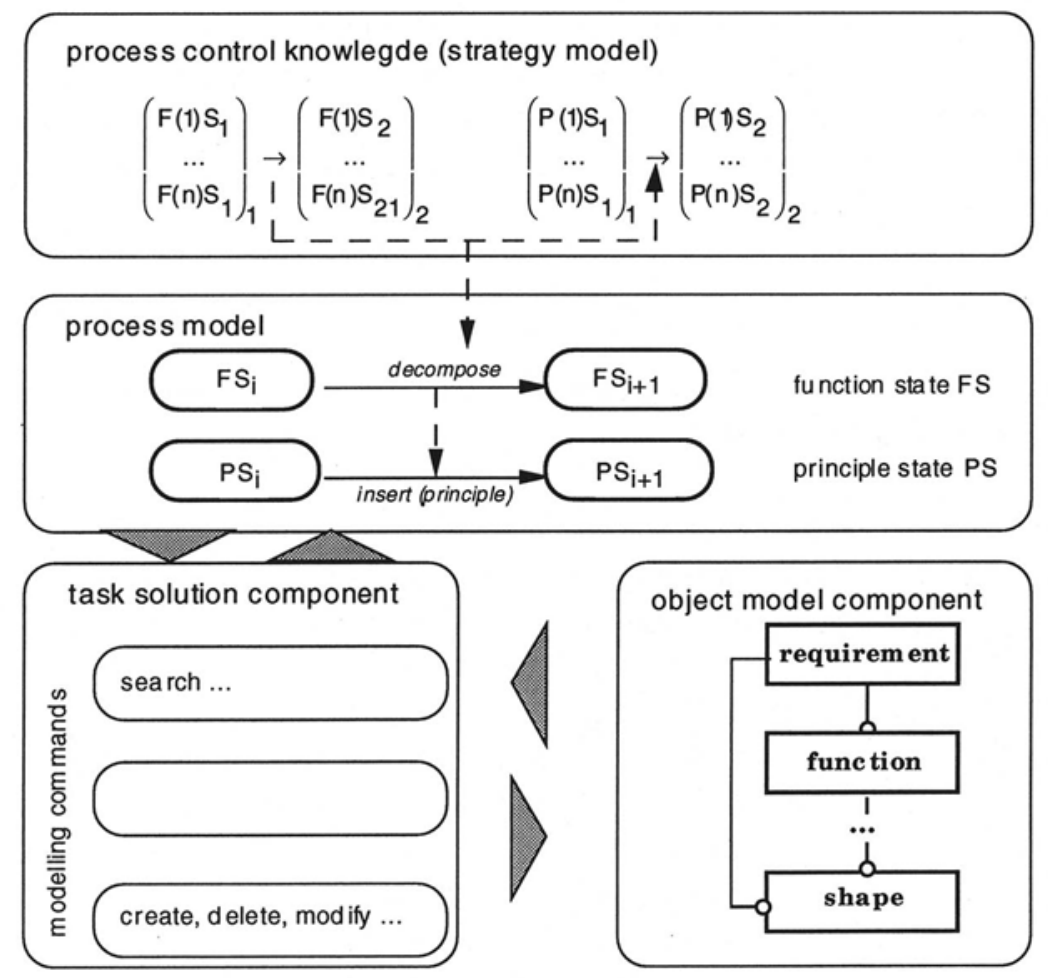

Figure 12. Architecture of the knowledge based CAD systems.

The third component of our system architecture is the "process model component". It contains a dynamic model of the design process. This dynamic model contains the objects of the object model. It contains also the different states in which the objects can find themselves. The dynamic aspect now describes the possible transitions between the different states of the objects and, as a very important point, by which actions these transitions are caused. The dynamic model is used by the process model component in order to compute the path of design steps leading to a description of the intended product.

Finally the fourth component of the system is the "process control component" which is responsible for the design path leading from the requirements specification of an intended product to its shape modeling. 
The process control component evaluates the status of the current design and computes on the basis of the process model the next design step which should be performed in order to transform a solution state $\mathrm{SS}_{\mathrm{i}}$ into a following state $\mathrm{SSO}_{\mathrm{i}+1}$. The information computed here contains on the one side the design object which should be manipulated in the next step, on the other side it contains the solution direction in which the design process should step forward. The process control component contains different general strategies which lead through the design process. The most important strategies which are modeled is on the one side the course through the design along the main functions of the intended product and on the other side the strategy "design along the functional flow" can be applied. In each case the process control component causes the modeling of the requirements of the intended product. After finishing the requirements specification, the functional modeling is initiated. When the functional structure of the system has been modeled, the designer chooses one strategy which seems to him to be an appropriate way for coursing through the design process. By the help of the chosen strategy, which is modeled in the system and together with the process model, the system calculates the design object which should be manipulated in the next step. Also the solution direction (see section 2) to be applied is chosen. So the process control component passes the reference to the design object and the solution direction to the task solution component which is responsible for manipulating the instantiated object model of the design.

It is understood that the general strategies currently contained in the process control component are not sufficient for completely controlling the design process. For that reason the research work is directed towards a learning design process controlling system. This additional approach takes the task dependent knowledge into account. This means the system has to learn the design steps for a specific task from the designer. Those steps have to be stored in combination with the respective solution states of the intended design in order to retrieve them when a design with similar requirements and functional structure has to be performed.

\subsection{THE DESIGN PROCESS MODEL}

Depending on the experience and skill, a designer chooses the appropriate path for the particular steps at different design stages. One important point for the development of an intelligent design system is to develop a model which describes this design process knowledge in a computable form. This means that an intelligent design system must support the designer in finding the right way through the design by navigating through the modeling space of design. For that reason the possible solution directions, applied to the objects of the respective modeling layers, described in section 2 , have to be 
modeled in a so called dynamic model of the design process. This model shows the dynamic behaviour of the design objects over time.

\subsection{THE DYNAMIC DESIGN OBJECT MODEL}

The design process model consists of two components. One is the dynamic model which describes exactly, thus in a computable form, the dynamic behaviour of the design objects during the design process. This means the states which an object can assume are modeled as well as the state transitions of the object. The second is the process control model, described after the dynamic model. The contents of a dynamic model are shown in Figure 13 and are described in the following.

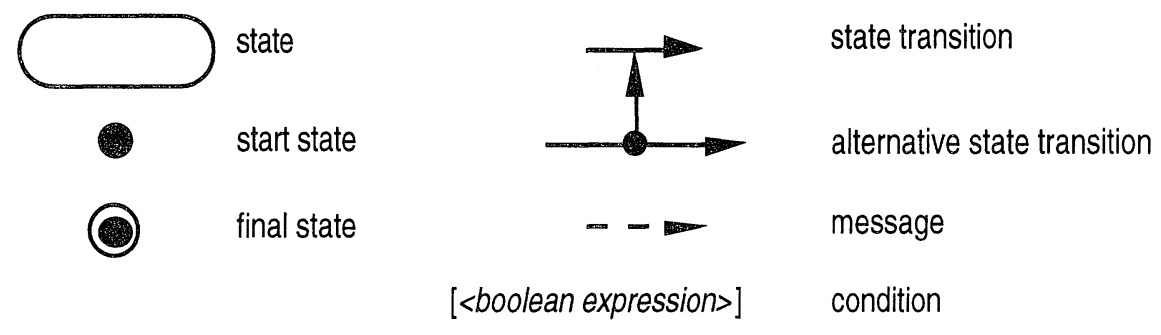

Figure 13. Symbols used in dynamic modeling.

Figure 13 shows the different symbols used in a dynamic model. The symbol "state" stands for the state which the modeled object can assume. An instantiation of the state, for example, can be detailed or varied. In the dynamic model this means, the object is in the state detailed or varied, respectively.

There are two other special states in the modeling method. These are the start state and the final state. The start state characterizes the object in its starting point before its first instantiation, whereas the object is in its final state when all attributes are defined and consolidated so that in this design step no further work will be done again on this object.

The arrows connecting two states symbolize the state transitions. A state transition is always caused by an action which designates this transition.

A special kind of state transition is the alternative state transition. This means that if the main action which designates the respective transition cannot be performed, the alternative transition caused by the corresponding alternative action is performed.

The last type of items which are used in dynamic modeling is the message. When an object reaches a state, which was specified in the model, a message can be sent in order to start an action. For that reason the dashed 
arrow which is the symbol for the message type always points from a state type to a state transition type.

In the case when two transitions which start from the same state are possible, the specification of conditions (symbolized by a rule enclosed in brackets) is necessary. So with the help of conditions it is possible to specify the transition which is only followed in the case in which the respective condition is fulfilled.

Figure 14 shows the dynamic model of the object general function. As the general function specification layer after the design methodology described in paragraph 2 , is the lowest layer in the concretion hierarchy, the design starts with the description of the general function of the intended product. When the requirements which the intended product has to fulfill are specified, the object General Function (GF) is in its initial state. Here the action initiate is performed. By initiation the object GF changes its state from the start state to the state defined. The two transitions starting at the state defined, symbolize that there are two possibilities for the next transitions. One is the transition to the state varied, the other is the transition to the state defined. The transition to the state varied is marked with the condition [GF.detailed $\neq$ NULL]. This means, this transition is only then followed if the instantiation of the object General Function has already been detailed before.

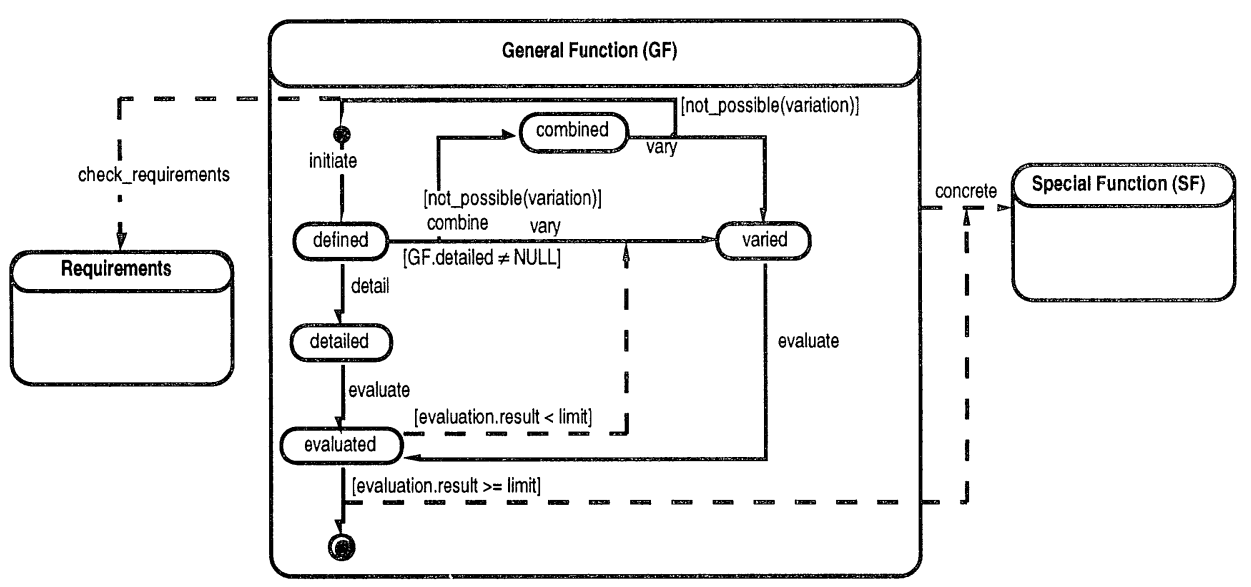

Figure 14. Dynamic model of the object "General Function".

In consequence, if General Function has not yet been detailed before, the transition to the state "detailed" is accomplished. The new state, detailed, is then reached by the action "detail". After detailing, the results of the action 
must be evaluated (how the evaluation is performed will be described in the following). By this the state "evaluated" is reached. The evaluation calculates a result (evaluation.result) which describes the quality of the found solution which was gained by the design step currently performed. The result of the evaluation is then compared with a certain limit. If the result is better than the limit, the final state the object general function is reached. This means the design for the general function is accepted. Here a message is sent in order to cause the sending of the message "concrete" to the object Special Function (SF). This means, the general function object is concreted to the corresponding special function.

If the result is worse than the limit, a message is sent to the transition vary. This message causes the execution of the variation of the general function object. In our model, the transition "vary" is modeled as an alternative transition. This means, if the intended variation can be performed, the state "varied" will be reached. Then the properties of the object will be evaluated in analogy to the object in the state detailed. If the variation cannot be executed (symbolized by the condition [not_possible(variation]), the alternative transition is started. This alternative causes the combination of the object so that the object will reach the state "combined".

When the object is in the state "combined", the transition vary is performed. In the case when a variation is not possible, no useable result has been reached in this design step. In consequence, the object general function returns into its start state, while the corresponding transition sends the message "check_ requirements" to the object requirements.

Similar to the dynamic behaviour of the general function object is the behaviour of the other objects which belong to the different modeling layers (see also Figure 15).

Figure 15 shows on overview of the dynamic behaviour of the objects which classify the different modeling layers in design. The internal dynamic behaviour of the objects shown (special function, physical principle, effective structure and embodiment) is very similar to the general function. The difference is that if no solution for the specified problem is found, the objects are abstracted to each next higher level of abstraction. This abstraction can be executed until the highest level, the general function modeling, is reached. Here an abstraction is no more possible. If no solution is found, as shown in Figure 14 the requirements have to be checked for correctness. If the intended design solution has to fulfill all the specified requirements, and no appropriate solution can be found, in consequence, the design project has to be stopped.

The process model represents all states, state transitions etc. which are described in the modeling space of design being fundamental for navigating through the design process. A special "path" for particular steps is not 
described in the dynamic design model but in the process control model in which knowledge of designers, their experience and skill is described. This process control model controls special state transitions and messages of the dynamic design model.

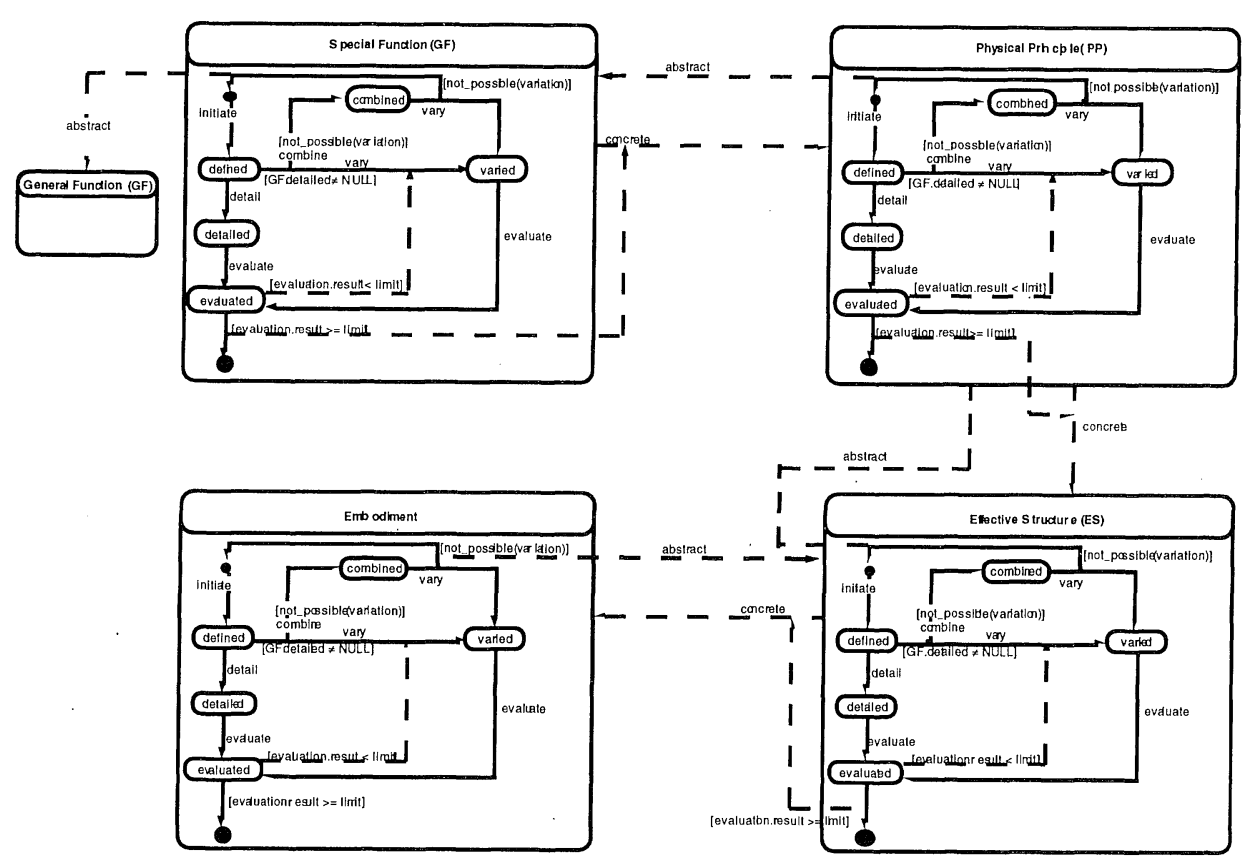

Figure 15: Overview of the dynamic model of the objects which describe the abstract modeling layers in design.

\subsection{THE PROCESS CONTROL MODEL}

The dynamic design model, described above, is responsible for the selection of the solution directions $i$. e. the modeling commands applied next to a given design object in a given design working space. This procedure can be called a "tactic" which is subordinated to a superior design strategy embodied by the process control model. So the process control model contains several design strategies which describe the design process on a higher level than the dynamic design model. These process control strategies are heuristics which describe a path leading to the solution of a design task. The way resulting by the strategies usually is not optimal and by that it does not lead directly to the intended design solution. Partially iteration cycles are necessary in order to improve already found solutions or to correct directions followed by mistake. Because of their task- 
independencies, process control strategies form the basis for a process control of an intelligent CAD system. This means they describe independently from the specific design task a path through the design leading to the intended task solution. The knowledge which is necessary for this task is based on the general design methodology. As example for such strategies "design along the flow of force, begin at the source" or "design along the main functions" can be mentioned. It is easy to see that these general, task independent strategies can show any path leading to the solution of the design task. But this path usually will not be optimal. In this context the term optimal means to design a product which fits optimally to the requirements specified applying possibly few design steps. Finding such an optimal path needs knowledge about optimal design processes depending on the respective design task. For that reason in our research work we try to develop a concept to acquire task dependent process knowledge from the designer in order to reuse it when a similar task appears.

\section{Conclusions and Future Work}

We have modeled and verified the dynamic model in a small application on the DIICAD product model. Modeling design solutions in design working spaces and saving these design solutions as solution patterns in the product model is possible. With design working spaces we find similar solution patterns for a given problem. This is realized by using a case-basedreasoning approach which is implemented on KEE and ACIS. At the moment we are able to do this for the requirements modeling (Kläger, 1993) and the functional (Huber, 1994) design stages in a top down approach and for solution patterns (Suhm, 1993) described in the mentioned $\mathrm{PhD}$ theses of Kläger (1993), Suhm (1993) and Huber (1994).

Modeling the design process in the described way is a promising approach. We have modeled the product life cycle and verified the approach in a small prototype. In the SFB346 (this is a special research area which is set up at the University of Karlsruhe by the German Research Community) a language has been developed to describe dynamic models. We consider it an important point that in the future basic research has to be done in developing a methodology to build dynamic models. Our next step will be to implement the process model as a whole (dynamic design model and process control model) which will be controlled by the process control model. Another step is to develop a methodology to gain design process knowledge from the designer by configuring so called process patterns which are analogously the same on the dynamic level as solution patterns (Suhm, 1993) on the static level. 


\section{References}

Benz, T.: 1990, Funktionsmodellierung als Basis zur Lösungsfindung in CAD-Systemen (Functional Modeling as a Basis for the Solution Finding in CAD Systems) Universität Karlsruhe (TH).

Birkhofer, H.: 1980, Analyse und Synthese der Funktion technischer Produkte (Analysis and Synthesis of the Function of Technical Products), Dissertation, TU Braunschweig.

Huber, R.: 1994, Wissensbasierte Funktionsmodellierung als Grundlage zur Gestaltsfindung in Konstruktionssystemen /(Knowledge based functional Modeling as Basis for the Shape Computing in Design Systems), Aachen: Shaker (Reihe Konstruktionstechnik), Zugl.: Karlsruhe, Univ., Diss., Institut für Rechneranwendung in Planung und Konstruktion (RPK).

Kläger, R.: 1993, Modellierung von Produktanforderungen als Basis für Problemlösungsprozesse in intelligenten Konstruktionssystemen (Modeling of Product Requirements as Basis for Problem Solving Processes in intelligent Design Systems) Aachen: Shaker, (Reihe Konstruktiostechnik), Zugl.: Karlsruhe, Univ., Diss., Institut für Rechneranwendung in Planung und Konstruktion (RPK).

Koller, R.: 1985, Konstruktionslehre für den Maschinenbau (Design Theory for the Mechanical Engineering), Springer-Verlag, Berlin.

Krumhauer, P.: 1974, Rechnerunterstützung für die Konzeptphase der Konstruktion (Computer Support for the Conceptual Phase of Design), Dissertation, TU Berlin.

Pahl, G. and Beitz, W.: 1994, Engineering Design, Springer-Verlag, Berlin.

Roth, K.-H.: 1994, Konstruieren mit Konstruktionskatatogen, Springer-Verlag Berlin.

Rutz, A.: 1985, Konstruieren als gesdanklicher Prozeß (Design as Intellectual Process) KM Lehrstuhl für Konstruktion im Maschinenbau TU München, Dissertation.

Suhm, A.: 1993, Produktmodellierung in wissensbasierten Konstruktionssystemen auf der Basis von Lösungsmustern (Product Modelling in Knowledge Based Design Systems on the Basis of Solution Patterns) Aachen: Shaker, (Reihe Konstruktionstechnik), Zugl.: Karlsruhe, Univ., Diss., Institut für Rechneranwendung in Planung und Konstruktion (RPK).

von Weizäcker, C. F.: 1971, Die Einheit der Natur-Studien. (The Unity of the Nature Studies), Hanser, Munich.

Wallace, K., Ball, B. and Tang, M-X.: 1995, AI in mechanical engineering design, in J. S. Gero and F. Sudweeks (eds.), Fourth Workshop on Research Directions for Artificial Intelligence in Design, University of Twente, Enschede, The Netherlands. 